\title{
Cogitare
Enfermagem
}

\section{EL CUIDADO DE SÍ Y LA ESPIRITUALIDAD EN TIEMPOS DE CONTINGENCIA POR COVID-19}

Raúl Fernando Guerrero Castañeda', Quetzalcóatl Hernández-Cervantes

\section{RESUMEN}

Objetivo: reflexionar sobre el autocuidado y la espiritualidad en tiempos de contingencia por COVID-19.

Desarrollo: ante el pronunciamiento global de la pandemia por COVID-19, en el mundo se han implementado distintas medidas de control, destacando el aislamiento físico voluntario a fin de contener la propagación del virus; así, el ser humano se enfrenta a un fenómeno poco cotidiano donde resalta el distanciamiento. Foucault, Heidegger y Watson contemplan conceptos elementales para reflexionar sobre la introspección y la espiritualidad como formas de auto cuidado, permitiéndole al individuo afrontar efectivamente el aislamiento, al mismo tiempo que fomentar su resiliencia y desarrollo humano.

Consideraciones finales: el cuidado espiritual que puede proveer la enfermería es un área que se convierte en el foco de interés para contribuir en la salud mental de las personas en aislamiento, es decir, una oportunidad para la promoción de salud.

DESCRIPTORES: Cuidado de Enfermería; Covid-19; Aislamiento Social; Espiritualidad; Filosofía.

COMO REFERENCIAR ESTE ARTIGO:

Castañeda RFG, I Hernández-Cervantes Q. El cuidado de sí y la espiritualidad en tiempos de contingencia por Covid-19. Cogitare enferm. [Internet]. 2020 [acesso em "colocar data de acesso, dia, mês abreviado e ano"]; 25. Disponível em: http://dx.doi.org/10.5380/ce.v25i0.73518.

${ }^{1}$ Enfermero. Doctor en Ciencias de Enfermería. Profesor de Departamento de Enfermería Clínica de la Universidad de Guanajuato. Celaya, Gto, México. (2)

${ }^{2}$ Psicólogo. Doctor en Psicología y Salud. Profesor investigador del Departamento de Ciencias de la Salud de la Universidad Iberoamericana Puebla. San Andrés Cholula, Puebla, México. 


\title{
O AUTOCUIDADO E A ESPIRITUALIDADE EM TEMPOS DE CONTINGÉNCIA POR COVID-19
}

\begin{abstract}
RESUMO
Objetivo: refletir sobre o autocuidado e a espiritualidade em tempos de contingência por COVID-19.

Desenvolvimento: a partir da declaração global da pandemia de COVID-19, diferentes medidas de controle foram implementadas em todo o mundo, destacando-se o isolamento físico voluntário para conter a propagação do vírus; assim, o ser humano enfrenta um fenômeno desconhecido, no qual o isolamento torna-se essencial. Foucault, Heidegger e Watson contemplam conceitos elementares para refletir sobre introspecção e espiritualidade como formas de autocuidado, permitindo que o indivíduo enfrente efetivamente o isolamento, promovendo, por sua vez, sua resiliência e desenvolvimento humano.

Considerações finais: o cuidado espiritual que pode ser oferecido a través da enfermagem é uma área que concentra o foco de interesse a fim de contribuir com a saúde mental de pessoas com deficiência, isto é, uma oportunidade para a promoção da saúde.
\end{abstract}

DESCRIPTORS: Cuidados de Enfermagem; Covid-19; Isolamento Social; Espiritualidade; Filosofia.

\section{SELF-CARE AND SPIRITUALITY IN TIMES OF CONTINGENCY DUE TO COVID-19}

\begin{abstract}
:
Objective: To reflect on self-care and spirituality in times of contingency due to COVID-19. Development: In view of the global declaration of the COVID-19 pandemic, different control measures have been implemented worldwide, highlighting voluntary physical isolation in order to contain the propagation of the virus; thus, mankind faces a very unusual phenomenon where distancing stands out. Foucault, Heidegger, and Watson contemplate elementary concepts to reflect on introspection and spirituality as types of self-care, allowing the individuals to effectively face isolation, while fostering their resilience and development as human beings. Final considerations: The type of spiritual care that Nursing can provide is an area which turns into the main interest in order to contribute in the mental health of the people in isolation, that is, an opportunity for the promotion of health.
\end{abstract}

DESCRIPTORES: Nursing Care; Covid-19; Social Isolation; Spirituality; Philosophy. 
Actualmente, se ha instaurado a nivel mundial una contingencia sanitaria, producto de la pandemia por COVID-19, que inicia a finales de 2019 y que actualmente cursa con una alta incidencia de $\operatorname{casos}^{(1)}$. Los gobiernos del mundo han implementado medidas sanitarias que exigen que las personas permanezcan en aislamiento voluntario u obligado. En México, el 20 de abril de 2020 la Secretaría de Salud declaró la Fase 3, lo que conlleva medidas estrictas de aislamiento y la protección de personas en condiciones vulnerables ${ }^{(2)}$.

El aislamiento como una forma de protección para reducir los contagios también trae consigo efectos en la salud mental. Por sí mismo es capaz de producir altos niveles de ansiedad, estrés, tristeza y otros síntomas que alteran la salud mental de las personas ${ }^{(3,4)}$. Aunado a ello, la noción de exposición y la posibilidad de contagio, la gran cantidad de información y exposición mediática, sumando el desequilibrio social y económico, hacen compleja esta problemática.

Esta pandemia ha dejado ver las diversas carencias en salud mental que la población tiene, y que a una proporción importante no le permite afrontar de manera saludable el aislamiento(5). Por ende, se ha evidenciado la necesidad de implementar estrategias de promoción de la salud mental que permitan a las personas desarrollar una resiliencia específica a esta situación y que ha tornado abrumadora ${ }^{(6)}$.

Es necesario reflexionar sobre las formas de autocuidado, que van más allá de lo físico, dentro de las diferentes manifestaciones del cuidado de sí también está la espiritualidad, vista como una forma de encuentro consigo mismo, con la naturaleza, en ocasiones asociada con alguna divinidad, y que puede incluir matices religiosos ${ }^{(7-10)}$. En este sentido, la espiritualidad como forma de cuidado, fomenta la introspección y dota al ser humano de la capacidad de contemplarse a sí mismo. Igualmente, esta forma de autocuidado facilita el desarrollo de estrategias resilientes, donde a través de sus creencias y prácticas le permite a la persona afrontar situaciones complejas y difíciles. En un sentido mínimo, el ideal de la espiritualidad supone para el individuo el poder lograr el equilibrio entre mente, cuerpo y espíritu $^{(11)}$.

Fenomenológicamente, Foucault aborda la espiritualidad como una forma de acción sobre la misma vida ${ }^{(12)}$, en acciones de cuidado de sí, un recurso que el ser humano encuentra para proporcionarse paz, bienestar y contemplación. Desde esta perspectiva integrada, el auto cuidado se constituye como la forma más elevada de conciencia personal, que promueve la trascendencia del ser humano. Cuidar el cuerpo entonces es esencial, pues es cuidar la conciencia y el espíritu, que sanan y dan sentido a la vida misma. En la espiritualidad como forma de cuidado, Heidegger aborda la ocupación de sí y la preocupación como las primeras formas de cuidado del ser humano(13), atendiendo la necesidad de la práctica espiritual para trascender; el cuidado de sí mismo desde la espiritualidad sería el camino para poder comprender las situaciones cotidianas y encontrar un sentido a lo vivido.

Añadiendo esta primera reflexión, entonces la espiritualidad que forma parte de las modalidades del cuidado humano de sí no es tanto una forma de resignación, sino una oportunidad de aprendizaje con un propósito particular, que coadyuva al establecimiento del sentido de vida para cada individuo. No obstante, para que esto suceda, resulta fundamental la contemplación de conexiones más allá de los fenómenos religiosos culturalmente establecidos en determinados contextos históricos. Por ejemplo, en el escenario mexicano y latinoamericano, este contexto está ligado a la práctica del catolicismo y el cristianismo, por lo que resulta pertinente ponderar los elementos de tal contacto religioso, así como determinadas creencias que pueden incluso no ser vividas plenamente en la vivencia actual de crisis sanitaria.

En esta reflexión sobre algunos elementos de la espiritualidad, desde el constructo del cuidado de sí, se identifican elementos como la trascendencia y la contemplación. Integradas, permitirían al personal de enfermería reflexionar sobre el propio proceso del 
cuidado espiritual, y a su vez desarrollar elementos de promoción de la espiritualidad en tiempos de crisis desde la reflexión disciplinar.

Por lo anteriormente expuesto, el propósito del presente artículo es reflexionar sobre el cuidado de sí, la espiritualidad, la contemplación y la trascendencia en tiempos de contingencia por COVID-19, teniendo como referentes algunos conceptos de Foucault, Heidegger y Watson.

\section{DESARROLLO}

El ser humano, absorto en la cotidianidad, no se contempla a sí mismo; en las ocasiones que sus formas de ser no le permitan plenitud, está continuamente pensando que el "ser" se limita a la actividad laboral, al cumplimiento de un papel, al desempeño de una acción. Identificamos así papeles temporales, de tal manera que cuando emerge el caos, la actividad laboral se suspende, se incrementa el papel de mamá o papá ante el cambio de la actividad escolar, el ser humano no encuentra más que crisis por no suspender lo cotidiano. En ese momento, la espiritualidad como un camino de búsqueda y de encuentro consigo mismo resulta viable y puede constituirse como una forma de cuidado con diferentes vías o estrategias.

Foucault ${ }^{(12)}$ señala respecto al cuidado de sí mismo una interrelación entre las diversificaciones de sus formas de ser, una preocupación de sí mismo que le provee mecanismos de equilibrio, mientras que Heidegger aparece con los modos de ser. Resultaría común que, en la cotidianidad, el ser humano se envuelve en conductas, pensamientos y relaciones efímeras, sin haber un interés por saber quién es, o por ser él mismo, sino por lo que acontece en el mundo para responder a éste como mejor le sea posible. Precisamente Heidegger sitúa la espiritualidad como parte de la intencionalidad de la vida, la noción de cuidarse busca el sentido a través de la espiritualidad como una forma de encontrar conocimiento y sentido(14).

La espiritualidad desde Foucault(12) responde a un acto de conocimiento por sí mismo y para sí mismo, de manera tal que el ser humano accede al conocimiento pleno de la verdad en un proceso de introspección y desarrollo humano, permitiéndole así lograr un auto conocimiento que le posibilitaría transformar su propia vida, pues manifiesta una relación de comprensión de sí. En este sentido, la espiritualidad como camino permitiría lograr una contemplación del ser y de su momento actual, a través de la reflexión ${ }^{(14)}$, entendida como una forma de auto revelación.

La espiritualidad entonces podría constituirse como un elemento de agencia en salud mental, que le otorga a la persona una comprensión del momento difícil, una reflexión sobre el aislamiento que vive de manera particular; un modo de análisis de la situación de salud. Vista así, la espiritualidad se convierte en una acción de cuidado a sí mismo, un cuidado que en palabras de Heidegger es per se una forma de comprensión ${ }^{(13)}$. Entonces, el aislamiento, lejos de ser un periodo de crisis, se convierte en una oportunidad de trabajo interior sobre lo que es y lo que hace.

Para Watson ${ }^{(15,16)}$, el cuidado es un acto que va más allá de lo físico y de las preocupaciones inherentes a los modos de presencia humana. Es decir, contempla las dimensiones de lo emocional y lo espiritual como parte del cuidado físico, por lo que la ciencia del cuidado tendría que estar promoviendo acciones que permitiesen el acercamiento -incluso doloroso- consigo mismo. Una forma de entender lo anterior es visualizar que como se actúa cotidianamente no necesariamente constituye un auténtico sentido personal. Ante lo anterior, se pondera la espiritualidad como una forma de cuidado; "crear un ambiente sutil de sanación energética en todos los niveles de conciencia, en la cual se realza la integridad, la belleza, la comodidad, la dignidad y la paz" (21:6).

El encerrarse en lo inusual, el estar en un ambiente cambiante y diferente, puede 
acarrear preocupación. Esta preocupación es la primera señal de adaptación, pues es el reconocer lo desconocido y el posible daño que pueda causar. Al respecto, Foucault ${ }^{(12)}$ identifica que la preocupación tiende a ser la primera respuesta del ser ante sus dudas existenciales. Primero aparece por una forma y ésta puede mantenerse alejada de lo que en realidad se conoce de sí mismo; en el momento actual, el aislamiento conllevaría a un enfrentamiento de la realidad propia del ser humano y de sus debilidades, aquéllas que incluso no contemplaba.

Dentro las formas de cuidado de sí, la espiritualidad fomenta la búsqueda de sí mismo que contempla incluso el alma, lo que Foucault aborda como la cuestión del uno mismo (heautou). "Ocuparse de uno mismo significa ocuparse de su alma: yo soy mi alma" (12:46). El alma resulta en un movimiento interno de sí mismo que se vuelve acción propia, distante de la concepción cristiana que alude a una dicotomía del ser entre cuerpo y alma como algo separado. Sin embargo, el alma es la acción en movimiento del ser, y sus manifestaciones expresadas en el cuidado de sí mismo, aludiendo al bienestar corporal, a la búsqueda de sentido y de la verdad; no como una razón única, sino de sentido personal.

Así, la contemplación del ser humano en estos momentos tendría que estarlo llevarlo a una plena búsqueda de la verdad, la verdad de sí mismo. Este proceso auto contemplativo se asociaría a un preguntarse continuamente quién es frente a sí mismo y hacia el mundo. Foucault menciona que "el sujeto debe, para acceder a la verdad, transformarse a sí mismo el algo distinto" (12:38). La espiritualidad se transforma en algo renovador, que le permite a la persona reconocer incluso aquello que para sí mismo es incómodo, y así acceder a nuevas formas de pensamiento y acción consigo mismo y su propósito de vida. Sin embargo, no habría que olvidar que antes de la conciencia espiritual, existe el cuidado como la razón de buscar las estrategias necesarias para hacer frente a las situaciones complicadas como las que se viven actualmente -la desesperación, la ansiedad, el aislamiento. Al respecto, Heidegger menciona que "la caracterización general de la temporalidad del cuidado, es la de hacer visible la impropiedad del Dasein en su específica temporalidad" (14:321).

El cuidado espiritual(17-19) per se es la contemplación del sentido de ser, para lo cual es necesario transitar por diversos niveles de la meditación espiritual; el movimiento del ser por la lucha de un encuentro consigo mismo. Para poderse encontrar es necesario conocerse, pero para conocerse es necesario entrar en contacto con sentidos más terrenales: el miedo, la incertidumbre, el vacío, la nostalgia, por mencionar algunos elementos. Cada movimiento del ser en este caminar debería contemplar principios básicos, producto de una reflexión del ser consigo mismo. La interacción con el alma en búsqueda de su verdad revelaría los valores más supremos de ser. El conocerse a sí mismo descubriría así la forma del cuidado del otro, un modo de procuración, un interés existencial por el otro, y resultando en la comprensión de sí mismo.

La ciencia de la enfermería y sus profesionales tienen ahora un modo de promoción del cuidado trascendental, mediante acciones comunitarias de promoción de salud desde el plano espiritual. En este periodo de caos, esta capacidad de agencia de la enfermería contribuiría a una organización vital diferente, orientada a poder contemplarse en su propio desarrollo y crecimiento humano; el cuidado también es trascendencia.

Algunas aplicaciones pueden ser el cuidar el ambiente en la medida de lo posible en momentos de reflexión personal, ambiente limpio, favorecer las conexiones humanas de quienes conviven en el confinamiento, crear una rutina personal que incluya un espacio para la contemplación, un espacio para la práctica de las creencias que favorezcan la fe y la esperanza ${ }^{(20,21)}$. La atención plena -o mindfulness- es otra práctica contemplativa bastante extendida y que puede ser un primer recurso para el fomento del cuidado de sí; es una práctica integrativa de la espiritualidad hacia la introspección ${ }^{(22)}$. Resulta oportuno identificar que otro tipo de intervención en enfermería es la empatía y la escucha activa, donde los cuidados en casa ayudarían a relaciones sanas, desde la comprensión de los momentos críticos del otro, y donde empatizar significa el contemplar al otro para comprender sus necesidades de cuidado.

El ser humano al ocuparse de sí mismo, es capaz de valorar el sentido propio del 
cuidado hacia el otro. Se puede traducir en empatía, incluyendo las relaciones consigo mismo, con los demás y con lo que considera divino ${ }^{(23)}$. El cuidado espiritual en la promoción de la salud mental por parte de enfermería resulta esencial. Si bien la lucha de algunas enfermeras y enfermeros se encuentra en los hospitales, otros y otras deben contemplar el cuidado en casa y la promoción de un crecimiento espiritual de los seres humanos, producto del cuidado de sí mismos en este periodo difícil.

\section{CONSIDERACIONES FINALES}

La enfermería puede intervenir en la promoción de la salud mental de las personas en confinamiento, particularmente desde las estrategias de auto cuidado que favorezcan la espiritualidad contemplativa del ser. Cuando éste se comprende a sí mismo a modo de cuidado, contempla al otro como un espejo y valora sus momentos más esenciales. En contraste, el desinterés no es una forma de cuidado, sino una constricción que parecería dejar encerrado al otro; por tal motivo, las acciones superficiales de cuidado no son un modo de ser en presencia, pero contribuyen a ella.

La espiritualidad es un área del ser humano que siempre ha estado ahí, pero que en este momento le permite a la persona desde su encierro la práctica de la contemplación en un nivel más esencial. Lo anterior le permite afrontar la situación compleja de aislamiento, y segundo, encontrarse consigo mismo, ayudándolo en su desarrollo y búsqueda del sentido de vida.

\section{REFERÊNCIAS}

1. World Health Organization. Coronavirus disease 2019 (COVID-19) Situation Report-72 HIGHLIGHTS [Internet]. 2020 [acceso en 28 abr 2020]. Disponible en: https://apps.who.int/iris/bitstream/ handle/10665/331685/nCoVsitrep01Apr2020-eng.pdf.

2. Secretaría de Salud. 110. Inicia la fase 3 por COVID-19 [Internet]. Inicia la fase 3 por COVID-19. 2020 [acceso en 28 abr 2020]. Disponible en: https://www.gob.mx/salud/prensa/110-inicia-la-fase-3-porcovid-19.

3. Huarcaya-Victoria J, Huarcaya-Victoria J. Consideraciones sobre la salud mental en la pandemia de COVID-19. Rev Peru Med Exp Salud Publica [Internet]. 2020 [acceso en 28 abr 2020]; 37(2). Disponible en: https://rpmesp.ins.gob.pe/index.php/rpmesp/article/view/5419.

4. Caballero-Domínguez CC, Campo-Arias A. Problemas de salud mental en la sociedad: un acercamiento desde el impacto del COVID 19 y de la cuarentena. Duazary. [Internet]. 2020 [acceso en 18 abr 2020]; 17(3):1-3. Disponible en: http://revistas.unimagdalena.edu.co/index.php/duazary/article/view/3467.

5. Medeiros AYBBV de, Pereira ER, Silva RMCRA, Dias FA. Fases psicológicas e sentido da vida em tempos de isolamento social por pandemia COVID-19 uma reflexão a luz de Viktor Frankl. Res Soc Dev. [Internet]. 2020 [acceso en 18 abr 2020] ;9(5): e122953131. Disponible en: http://dx.doi.org/10.33448/ rsd-v9i5.3331.

6. Ibáñez-Vizoso JE, Alberdi-Páramo Í, Díaz-Marsá M. Perspectivas Internacionales en Salud Mental ante la pandemia por el nuevo coronavirus SARS-CoV-2. Rev Psiquiatr Salud Ment. [Internet]. 2020 [acceso en 18 abr 2020]. Disponible en: https://dx.doi.org/10.1016\%2Fj.rpsm.2020.04.002.

7. Ferreira AG de C, Duarte TM de M, Silva AF da, Bezerra MR. Concepções de espiritualidade e religiosidade e a prática multiprofissional em cuidados paliativos. Rev Kairós Gerontol [Internet]. 2015 [acceso en 23 abr 2020]; 18(3):227-44. Disponible en: http://revistas.pucsp.br/index.php/kairos/article/ view/27054/19186. 
8. Vargas-Escobar LM, Guarnizo-Tole M, Tovar-Pardo MF, Rojas-Pérez CM. Tendencias de investigación en torno al cuidado espiritual de enfermería: Revisión de la literatura. Rev Investig Salud Univ

Boyacá. [Internet]. 2019 [acceso en 23 abr 2020]; 6(1):145-69. Disponible en: http://dx.doi. org/10.24267/23897325.304.

9. Weathers E, McCarthy G, Coffey A. Concept analysis of spirituality: an evolutionary approach. Nurs Forum [Internet]. 2016 [acceso en 15 abr 2020];51(2):79-96. Disponible en: http://doi.wiley.com/10.1111/ nuf.12128.

10. Lavorato Neto G, Rodrigues L, Silva DAR da, Turato ER, Campos CJG. Spirituality review on mental health and psychiatric nursing. Rev. Bras. Enferm. [Internet]. 2018 [acceso en 01 mayo 2020]; 71(Suppl 5):2323-33. Disponible en: https://doi.org/10.1590/0034-7167-2016-0429.

11. Norman V, Rossillo K, Skelton K. Creating healing environments through the theory of caring. AORN J. [Internet]. 2016 [acceso en 16 abr 2020]; 104(5):401-9. Disponible en: https://doi.org/10.1016/j. aorn.2016.09.006.

12. Foucault M. Hermenéutica del sujeto. Madrid: Ediciones de la Piqueta; 1994.

13. Heidegger M. El ser y el tiempo. México: Fondo de Cultura Económica; 2015.

14. Heidegger M. Fenomenologia da vida religiosa. Petrópolis, RJ; 2010.

15. Watson J. Nursing. The Philosophy and Science of Caring. Boulder: University Press of Colorado; 2008.

16. Watson J. Human Caring Science. A Theory of Nursing. Boston: Jones and Bartlett; 2011.

17. Pinedo MT, Jiménez J. Cuidados del personal de enfermería en la dimensión espiritual del paciente. Revisión sistemática. Cult los Cuid. [Internet]. 2017 [acceso en 16 abr 2020];21(48): 110-8. Disponible en: https://doi.org/10.14198/cuid.2017.48.13.

18. Menezes TM de O. Spiritual dimension of care in health and nursing. Rev baiana enferm [Internet]. 2017 [acceso en 16 abr 2020]; 31(2):e22522. Disponible en: https://portalseer.ufba.br/index.php/ enfermagem/article/view/22522/14623.

19. Bernard M, Strasser F, Gamondi C, Braunschweig G, Forster M, Kaspers-Elekes K, et al. Relationship between spirituality, meaning in life, psychological distress, wish for hastened death, and their influence on quality of life in palliative care patients. J Pain Symptom Manage. [Internet]. 2017 [acceso en 16 abr 2020]; 54(4):514-22. Disponible en: https://doi.org/10.1016/j.jpainsymman.2017.07.019.

20. Sitzman K, Watson J. Caring science, mindful practice: implementing Watson's human caring theory. New York: Springer Publishing Company; 2018.

21. Tonin L, Nascimento JD do, Lacerda MR, Favero L, Gomes IM, Denipote AGM. Guide to deliver the elements of the Clinical Caritas Process. Esc Anna Nery. [Internet]. 2017 [acceso en 16 abr 2020];21(4): e20170034. Disponible en: https://doi.org/10.1590/2177-9465-EAN-2017-0034.

22. Cebolla A, Enrique A, Alvear D, Soler J, García-Campayo J. Psicología positiva contemplativa: integrando mindfulness en la psicología positiva. Papeles del Psicólogo. [Internet]. 2017 [acceso en 16 abr 2020];38(1):12-8. Disponible en: https://doi.org/10.23923/pap.psicol2017.2816.

23. Waldow VR. Cuidar de sí, cuidar del otro, cuidar del todo: implicaciones para la salud y enfermería. Enfermería Cuid Humaniz [Internet]. 2016 [acceso en 18 abr 2020]; 2(1):53-6. Disponible en: http:// revistas.ucu.edu.uy/index.php/enfermeriacuidadoshumanizados/article/view/783. 
Recebido: 05/05/2020

Finalizado: 02/06/2020

Editora associada: Luciana Puchalski Kalinke

Autor Correspondente:

Raúl Fernando Guerrero Castañeda

Universidad de Guanajuato

Ing. Javier Barros Sierra 201, 38140, Celaya, Gto, México

E-mail: ferxtom@hotmail.com

Contribuição dos autores:

Contribuições substanciais para a concepção ou desenho do estudo; ou a aquisição, análise ou interpretação de dados do estudo - RFGC, QHC

Elaboração e revisão crítica do conteúdo intelectual do estudo - RFGC, QHC

\section{(c) (1)}

Este obra está licenciado com uma Licença Creative Commons Atribuição 4.0 Internacional. 